\title{
An MLP-Aware Leakage-Free Memory Controller
}

\author{
Andrew Vuong \\ University of Utah \\ Salt Lake City, Utah \\ andrewv@cs.utah.edu \\ Meysam Taassori \\ University of Utah \\ Salt Lake City, Utah \\ taassori@cs.utah.edu
}

\author{
Ali Shafiee \\ University of Utah \\ Salt Lake City, Utah \\ shafiee@cs.utah.edu \\ Rajeev Balasubramonian
University of Utah
Salt Lake City, Utah
rajeev@cs.utah.edu
}

\begin{abstract}
Timing channels can be exploited to leak information between two virtual machines running on a shared server. Indeed, cache timing channels are important components in the Spectre attack. A shared memory controller can also be leveraged to establish a timing channel. Recent efforts have designed memory controllers that eliminate such timing channels, but that incur throughput penalties of over $2 \times$. This paper advances the state-of-the-art by better matching the memory controller policies to the memory-levelparallelism (MLP) needs of typical applications. Our new memory controller improves upon the performance of the state-of-the-art policy by $14 \%$, while eliminating memory controller timing channels.
\end{abstract}

\section{CCS CONCEPTS}

\section{-Security and privacy $\rightarrow$ Hardware-based security protocols;}

\section{KEYWORDS}

Security, memory controllers, information leakage, timing channels

\section{ACM Reference Format:}

Andrew Vuong, Ali Shafiee, Meysam Taassori, and Rajeev Balasubramonian. 2018. An MLP-Aware Leakage-Free Memory Controller . In HASP '18: Hardware and Architectural Support for Security and Privacy, June 2, 2018, Los Angeles, CA, USA. ACM, New York, NY, USA, 7 pages. https://doi.org/10.1145/3214292.3214296

\section{INTRODUCTION}

Cloud platforms allow consumers to manage their computation and storage at low cost. These consumers also demand high levels of security and privacy for their sensitive data/computations, e.g., electronic health records. To a large extent, low cost is achieved because cloud operators dynamically schedule many workloads, belonging to different clients, on shared hardware. Each client or

Permission to make digital or hard copies of all or part of this work for personal or classroom use is granted without fee provided that copies are not made or distributed for profit or commercial advantage and that copies bear this notice and the full citation on the first page. Copyrights for components of this work owned by others than ACM must be honored. Abstracting with credit is permitted. To copy otherwise, or republish, to post on servers or to redistribute to lists, requires prior specific permission and/or a fee. Request permissions from permissions@acm.org.

HASP '18, June 2, 2018, Los Angeles, CA, USA

(C) 2018 Association for Computing Machinery.

ACM ISBN 978-1-4503-6500-0/18/06 . \$ \$15.00

https://doi.org/10.1145/3214292.3214296 workload may be assigned its own Virtual Machine (VM), thus isolating and protecting each client. However, when VMs execute on shared hardware, information side channels do exist between the VMs. For example, if two VMs share a hardware cache, the latencies and miss rates experienced by one VM can betray the cache behavior of the other VM. Such a timing channel has been exploited by Ristenpart et al. [10] to crack passwords in an Amazon cloud infrastructure, in spite of various noise and real-world phenomena.

The recent Spectre attack [8] relied on cache timing channels to learn the secret-dependent footprints left behind by speculative execution. A cache timing channel can be eliminated with techniques like cache partitioning [16, 17], which exists in some commercial hardware [9]. But an application's secrets can be leaked through a variety of side channels, including through shared hardware structures such as the memory controller [14] and branch predictor [4]. Modern processors do not currently incorporate defenses against memory controller timing channels. This is an important vulnerability that can be exposed by attacks styled after Spectre.

A concrete attack through the memory controller was demonstrated by Wang et al. [14]. They show that the memory intensity of a VM can correlate with the number of $1 \mathrm{~s}$ in the private key of an RSA decryption algorithm. Thus, by monitoring the memory intensity of a co-scheduled VM, an attacker VM can narrow the search space and quickly estimate private keys used by co-scheduled VMs.

Another important exploitation of memory-based timing channels is the establishment of a covert channel. Consider a cloud infrastructure set up by a hospital that includes electronic patient health records. The VMs used by the hospital may use state-of-theart firewalls and encryption to protect their sensitive data. But they may use untrusted third-party software, e.g., a document reader, that has full access to unencrypted data and serves as a trojan. Because of the firewalls, the third-party trojan software can't explicitly send sensitive data outside the firewall. But by establishing a covert channel, it can leak information to another VM that is coscheduled on the same hardware. In short, the third-party software can modulate the memory traffic rate, which is detected by the coscheduled VM, thus communicating 1s (high traffic) and 0s (low traffic) to the co-scheduled VM. Recent work by Hunger et al. [5] has demonstrated memory-based covert channels that can leak information between threads at a high rate of $500 \mathrm{Kbps}$.

Therefore, to protect a sensitive cloud application, it is important to close the timing channel that exists within the memory system or memory controller. In other words, it is important to 
design a memory controller where the memory latencies experienced by one VM are independent of the memory activity of other co-scheduled VMs.

A few recent papers have designed such memory controllers. Wang et al. [14] introduced the first leakage-free memory controller. They leverage the concept of Temporal Partitioning (TP) that essentially allocates the memory controller to one VM at a time in round-robin fashion. During its turn to use the memory controller, a VM first issues memory requests, then enters a dead time when no new requests are issued. Thanks to the dead time, the VM's requests do not pose resource conflicts with requests issued by the next VM. While this eliminates leakage between VMs, it introduces significant performance slowdown. This is because of bandwidth underutilization and long queuing delays stemming from a VM's long wait for its turn.

A follow-up project by Shafiee et al. [12] improved upon the performance of the TP design. It introduced a Bank Triple Alternation (BTA) mechanism that reduced the turn lengths for each VM. This approach imposes constraints on the banks that can be accessed in each turn, thus ensuring that accesses by one VM do not conflict with accesses by another VM.

Wang et al. [15] then introduced a solution, SecMC-NI, that represents the state-of-the-art in leakage-free memory controllers. SecMC-NI grows the turn length for each VM, but imposes scheduling constraints to maximize the memory requests issued in that turn. However, while better than prior work, this design only achieves $48 \%$ of the throughput achieved by a non-secure memory controller, i.e., the slowdown is significant and the room for improvement remains high.

In this work, we build upon the state-of-the-art in two ways. We first modify SecMC-NI with additional constraints similar to BTA. This helps reduce the turn length. It is also a better match for the memory-level parallelism (MLP) exhibited in most applications. Second, we observe that such constrained memory controller policies work better when requests are spread across many ranks. To promote such spreading, we employ a memory system that employs many rank subsets $[1,19]$. Our techniques bring the throughput levels closer to the non-secure baseline, while being implemented entirely in hardware and not increasing the burden on the OS.

\section{BACKGROUND}

\subsection{Threat Model}

As summarized in Section 1, we are targeting a cloud infrastructure where a single socket executes multiple different and unrelated VMs. These VMs may belong to different clients and do not trust each other. In fact, one of these VMs may be malicious and is attempting to extract information from other VMs. It does this by performing periodic memory accesses and recording average latencies for those accesses. Because of contention for the shared memory controller, these latencies reflect the memory intensity of co-scheduled VMs. This information leak either (i) betrays access patterns of co-scheduled threads, e.g., revealing the content of keys [10], or (ii) can be used to establish a covert channel between VMs. As described in Section 1, a covert channel can be used by a trojan application in one VM to betray secrets to a cohort in another VM [5].

Note that such timing channel attacks are performed without compromising the OS and without requiring physical access to the hardware. It can be performed by a remote user simply by asking the cloud operator to execute the user's VM.

There may be several side channels in a system, e.g., timing channels in a shared cache, timing channels in a branch predictor, leakage through addresses visible on the memory bus, etc. To fully protect a VM, each of these side channels will have to be closed with piecemeal solutions. Therefore, the solutions in this paper that address the timing side channel in the memory controller must be combined with other orthogonal solutions, e.g., cache partitioning [16].

\subsection{Memory Controller Basics}

A memory controller manages the memory modules connected to a single memory channel. A channel is connected to multiple ranks and each rank is itself partitioned into multiple banks. Many timing parameters dictate when operations can be issued to each rank and bank. For a detailed rundown of relevant timing parameters, please refer to the descriptions in prior work $[12,14]$. Here, we'll focus on the bottomline timing constraints imposed by those parameters.

The memory controller can issue a request to one rank, and follow it with a request to a different rank (so that both requests can be performed in parallel). For the DRAM simulation parameters described in Section 4, requests to different ranks can be issued with a minimum gap of 6 memory cycles. Shafiee et al. [12] show that if two accesses to different ranks are issued with a 7-cycle gap, they are guaranteed to not interfere with each other, regardless of whether the accesses are reads or writes.

In a similar vein, the memory controller can also issue requests to different banks (either in the same rank or in different ranks) in parallel. Shafiee et al. consider all combinations of reads/writes and various timing parameters to show that requests to different banks will never interfere if they are separated by 15 cycles. Further, two requests to the same bank must be separated by 43 cycles so that the first access has enough time to complete (this assumes the worst case of a row buffer miss).

Based on the above discussion, we proceed with the following rules of thumb to ensure that two consecutive memory requests from different VMs do not compete for the same resources:

- Two accesses to different ranks must be separated by 7 cycles.

- Two accesses to different banks in a rank must be separated by 15 cycles.

- Two accesses to the same bank must be separated by $43 \mathrm{cy}-$ cles.

By following these rules, the memory controller can create a schedule of memory accesses such that two VMs do not interfere with each other and are not vulnerable to memory timing channels.

Note that most high-performance processors have multiple DDR3/DDR4 memory channels. Since each channel is independently controlled, we will focus on a single channel for most of our discussion. 


\subsection{Temporal Partitioning [14] and Fixed Service [12]}

The first attempt at eliminating timing channels in the memory controller uses a policy referred to as Temporal Partitioning (TP) [14] A VM is assigned a turn for a number of cycles (the turn length); memory requests are initiated in the early part of the turn; the VM does not initiate any requests in the last 43 cycles of the turn (referred to as dead time). This guarantees that all memory requests have been completed before the next VM begins its turn. As a result, the next VM views the memory system as a clean slate and its memory latencies are independent of the memory activity exhibited by the previous VM, i.e., timing channels are eliminated.

Shafiee et al. [12] create a deterministic triple-alternation schedule to reduce the dead time (bank triple alternation - BTA). They require that a VM, during a turn, issue requests to (say) bank-ids that are multiples of 3, i.e., bankid modulo $3=0$. In the next turn, the next VM must issue requests to banks where bankid modulo $3=1$. In the turn after that, the next VM issues requests to banks where bankid modulo $3=2$, and so on. By grouping banks in this manner and imposing restrictions on which banks can be accessed in a turn, the memory controller guarantees that consecutive turns will never access the same banks. This makes it easier to create a non-conflicting schedule of memory accesses without a long dead time. Shafiee et al. show that dead time can be reduced to 15 cycles while ensuring that the second VM's accesses are independent of activity in the first VM.

Figure 1 shows an example schedule for both TP and BTA. Note that both of these works recommend issuing a single request per turn to keep the turn length short and not have long queuing delays. Both works also show that if the OS can map each VM to its own set of ranks or banks, it is easier to create non-conflicting schedules and improve performance. However, such approaches are not palatable because of the higher OS complexity, the impact on other cloud management policies, and the relatively small number of ranks per channel. We will therefore only consider techniques in this paper that can be implemented entirely in hardware.
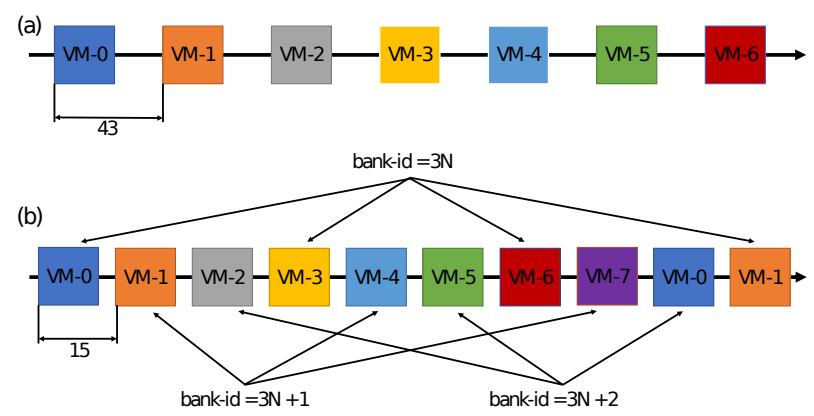

Figure 1: Example schedule for (a) TP and (b) BTA

\subsection{SecMC-NI $(3 R / 2 B)[15]$}

In recent work, Wang et al. [15] introduced the state-of-the-art solution, SecMC-NI, with examples shown in Figure 2. Wang et al. show that throughput can be increased by giving a VM a long enough turn to drain multiple requests, by using a short dead time, and by imposing some scheduling constraints that prevent resource conflicts across VMs.

Consider the example shown in Figure 2. A VM is given a 43cycle turn. Within that turn, the VM gets an opportunity to issue 6 memory requests -2 requests each from 3 different ranks. All 6 requests are sent to different banks. A deterministic conflict-free schedule is set up by interleaving requests to the three ranks, as shown in Figure 2. Following the rules of thumb described in Section 2.2, consecutive requests to different banks in the same rank are separated by more than 15 cycles; consecutive requests to different ranks are separated by at least 7 cycles.

In the first turn, VM-0 decides to issue requests to ranks R-3, R-6, and R-7 (since those ranks have the most pending requests). Accordingly, two requests to R-3, two requests to R-6, and one request to R-7 are placed in the six issue slots (one slot is not utilized). In the second turn, VM-1 decides to issue requests to ranks $\mathrm{R}-1, \mathrm{R}-4$, and R-2; it is able to fill all 6 slots. Note that in the two slots assigned to R-4, requests to banks B-3 and B-6 were issued. In the third turn, VM-2 decides to issue requests to ranks R-4, R-5, and R-6. The R-4 requests are to banks B-1 and B-6. Because of the schedule that was adopted for VM-1, VM-2 is forced to delay its access of B-6. This is done to preserve the 43-cycle gap between two accesses to the same bank. Because the banks being accessed in a turn are unique, we are guaranteed to find a correct conflict-free schedule for every turn, in spite of the constraints imposed by the schedule in the previous turn. Thus, a given VM is able to issue any requests it desires in a turn (while conforming to the rule that requests are to different banks in three ranks), regardless of what other VMs are doing.

With the above policy, the schedule may reveal what the other thread was doing. For example, if the previous VM repeatedly accessed rank R-5, the current VM's requests to R-5 typically happen in the later part of its turn. To eliminate this leakage, a VM should be oblivious to the schedule within its turn, i.e., it should not realize that R- 5 accesses are later than others. This is enforced by waiting until the end of the VM's turn to return all its requests en masse.

To make the subsequent discussion more intuitive, we will refer to the SecMC-NI policy as $3 R / 2 B$, i.e., a turn schedule that accesses 2 banks each in 3 different ranks.

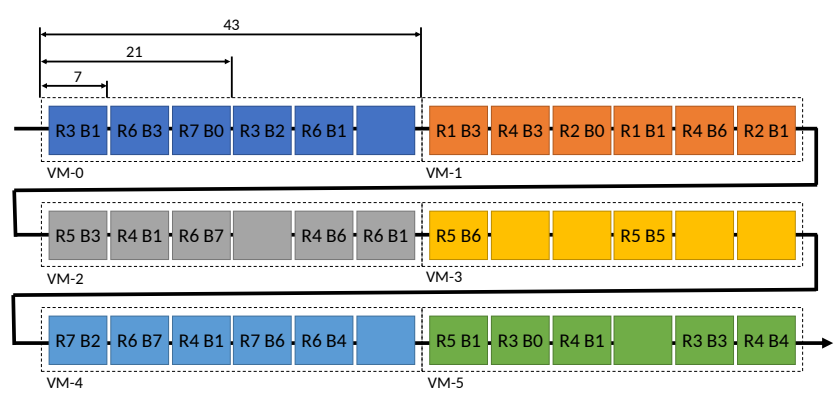

Figure 2: SecMC-NI example schedule. 


\section{PROPOSED MEMORY CONTROLLER POLICIES}

\subsection{Drawbacks of SecMC-NI}

As shown later in Section 5, the state-of-the-art SecMC-NI (or 3R/2B) solution achieves only $48 \%$ of the throughput achieved by a constraintfree non-secure baseline. If the VM does not have 2 accesses each to three different ranks, it will under-utilize the six available slots. Figure 3 quantifies this phenomenon.

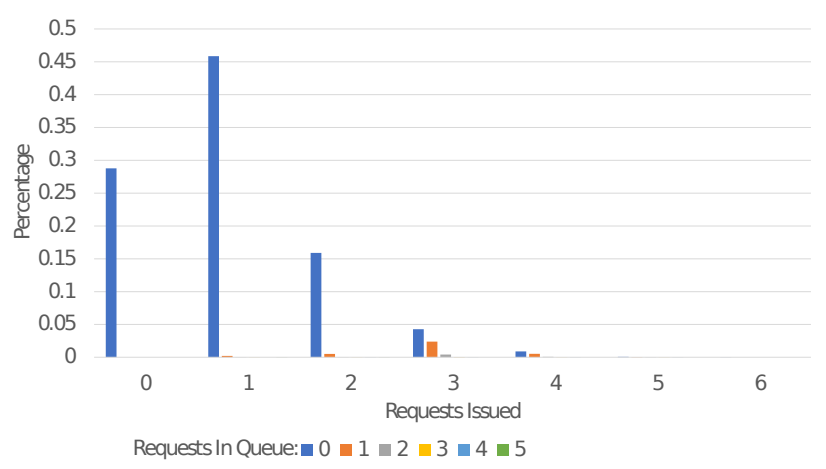

Figure 3: Histogram showing per turn stats for the baseline $3 R / 2 B$. Each cluster on the $X$-axis represents the number of requests issued in a turn; each bar within a cluster represents the number of requests that got left behind in the queue.

These results are for simulations with $8 \mathrm{VMs}, 8$ ranks, and a turn length of 43 cycles. Figure 3 shows a histogram to indicate the common scenarios encountered during a turn. Each cluster on the $\mathrm{X}$-axis represents a count of the requests that were issued in a turn; each bar in a cluster represents the number of requests that were left behind in the queue. We observe that in the most common scenario (occurs in $45 \%$ of all turns) 1 request is issued in a turn and 0 requests are left behind in the queue. This is indicative of the low degree of MLP in our benchmark suite. In fact, in nearly $90 \%$ of all turns, the benchmark has less than 2 pending requests. This indicates that a turn length that accommodates six issue slots will often be under-utilized. Overall, we observe that $82 \%$ of all issue slots in the $3 \mathrm{R} / 2 \mathrm{~B}$ scheduler go unutilized. Further, the long 43-cycle turn length also increases the wait time for each VM.

Table 2 quantifies the per-benchmark MPKI (last level cache misses per thousand instructions), which ranges from 0.2 to 30.5 . This range of MPKIs is similar to the ranges observed in other published workload characterizations $[6,11]$. For most out-of-order processors that have a window size under 200, the typical number of outstanding memory accesses is less than a handful for even the most memory-intensive benchmarks.

\subsection{Ranked Triple Alternation (RTA)}

The observations in the previous sub-section indicate that a $3 \mathrm{R} / 2 \mathrm{~B}$ baseline schedule leads to under-utilization because an application typically has low MLP. Two accesses to the same bank must be separated by 43 cycles; by having a 43-cycle turn length, the requests issued in one turn are not influenced by the requests issued in the previous turn. But a 43-cycle underutilized turn implies that applications have long wait times until they receive their next turn.

Our goal here is to shrink the turn length to improve utilization and reduce wait times. But if a shorter turn length is used, the banks touched in one turn are not free to receive new requests from the next turn, thus leaking information from one VM to the next. To prevent consecutive turns from touching the same banks, we introduce deterministic scheduling constraints that are applicationindependent. These scheduling constraints leverage the same concept used in BTA, but applied at the rank level.

We introduce Rank Triple Alternation (RTA), which shrinks the turn length to 14 cycles and allows a VM to issue 2 requests to 2 different ranks in its turn, with the added constraint that rank-id $\bmod 3=$ turn-id $\bmod 3$. In other words, in turn 0 , a VM is allowed to touch two different ranks with rank-ids that are both multiples of three; in turn 1, the next VM is allowed to touch two different ranks with rank-ids of the form $3 \mathrm{~N}+1$, and so on. After every 3 turns, a one-cycle bubble is introduced so that potential accesses to the same bank are separated by at least 43 cycles. An example of RTA is shown in Figure 4. RTA is also more effective than BTA because the gap between consecutive requests is 7 cycles in RTA and 15 cycles in BTA.

To ensure that a VM can touch different ranks in different turns, the total number of VMs should not be a multiple of 3 . If the number of co-scheduled sensitive VMs is a multiple of 3, the OS can introduce a dummy VM or use Rank Quad Alternation.

We also observe that RTA can issue two requests to the same rank in a turn as long as those two requests are of the same type (both reads or both writes) and are sent to different banks. Note that all of our schedulers employ an XOR address mapping policy to help evenly distribute requests across all ranks $[12,18]$.

\subsection{Rank Subsetting}

The proposed RTA scheduler does not improve performance when two requests are scheduled to different rows in the same bank. This bank-level conflict can cause delays because the second access must wait several turns before being issued. In general, the RTA scheduler has more options when requests are scattered across several banks and ranks. To promote such parallelism, we propose leveraging the concept of rank subsets [1,19]. Rank subsets deviate from the DDR standard. They require creating a custom DIMM, as is done by certain vendors, e.g., in Power8 systems [13]. Rank subsets, while more expensive than commodity DIMMs, are useful for several reasons - they improve parallelism and reduce energy per access. We make the argument here that rank subsets are also useful in alleviating some of the constraints in leakage-free memory controllers.

Rank Subsetting offers higher memory parallelism by forming smaller parallel ranks. This increases the number of ranks and banks. However, forming smaller parallel ranks increases the data transfer time and reduces the size of the row buffer. The data transfer time, tBURST, increases from 4 cycles to 8 cycles when a rank is partitioned into 2 sub-ranks (referred to as 2-way rank subsetting). We repeated the mathematical analysis of Shafiee et al. [12] for the new timing parameters imposed by rank subsets. This yields new 


\begin{tabular}{||c|c||}
\hline \multicolumn{2}{||c||}{ MPKI } \\
\hline \hline mcf & 30.505 \\
\hline astar & 2.584 \\
\hline omnetpp & 15.222 \\
\hline libquantum & 16.203 \\
\hline xalancbmk & 14.003 \\
\hline dealII & 0.236 \\
\hline bwaves & 0.254 \\
\hline gromacs & 2.584 \\
\hline gobmk & 0.599 \\
\hline sjeng & 0.529 \\
\hline hmmer & 0.902 \\
\hline
\end{tabular}

Table 2: MPKI for SPEC2k6 workloads.

is known to suffer from many bank conflicts. Such bank conflicts are more problematic in RTA given that the scheduler already imposes more constraints, e.g., only allowing requests to ranks that are multiples of 3 in a given turn.

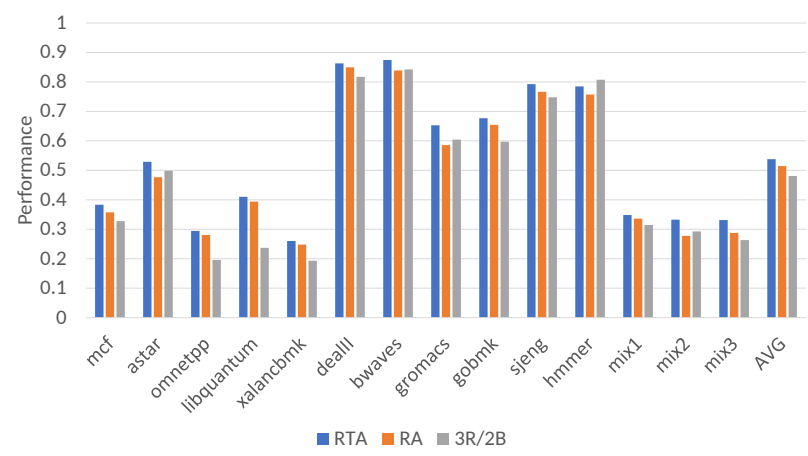

Figure 5: Performance comparision between baseline 3R/2B, RA and RTA. Performance is normalized to a baseline nonsecure scheduler.

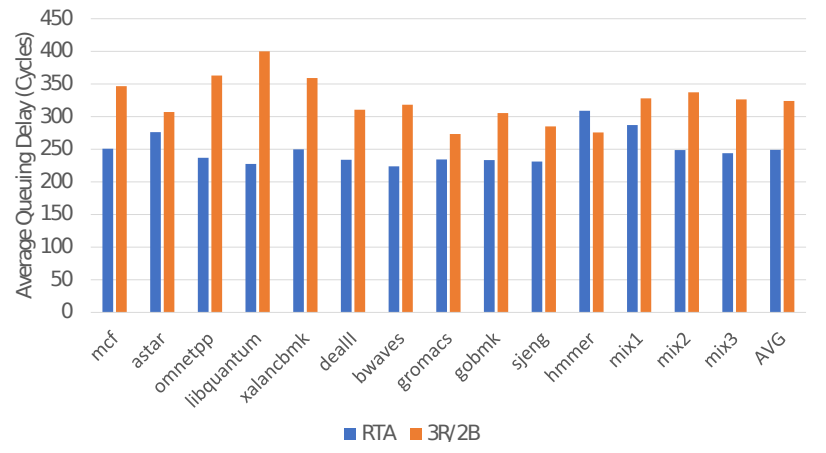

Figure 6: Average queueing delay of requests for RTA and $3 R / 2 B$.

\subsection{Performance with Rank Subsetting}

Next, we show the impact of rank subsetting on $3 \mathrm{R} / 2 \mathrm{~B}$ and on RPA, which is the alternation scheduler adapted for rank subsets. We will first consider 2-way rank subsets, i.e., each rank in our baseline is partitioned into two. We normalize all results against the non-secure baseline without rank subsets. In Figure 7, we see that rank subsets worsen performance for $3 \mathrm{R} / 2 \mathrm{~B}$ because they increase the turn length. However, the use of rank subsets with RPA is able to accommodate relatively short turns. The short turns and the fewer conflicts from rank subsets are able to offset the longer gaps between consecutive requests. Overall, we see that rank subsets and RPA are able to improve upon the performance of RTA by $3 \%$. While this alone is not a large enough benefit to create custom non-DDR-compliant DIMMs, it adds to the arguments in favor of DIMMs that employ narrow ranks: lower energy, higher parallelism, and now better tolerance to the constraints imposed by leakage-free schedulers.

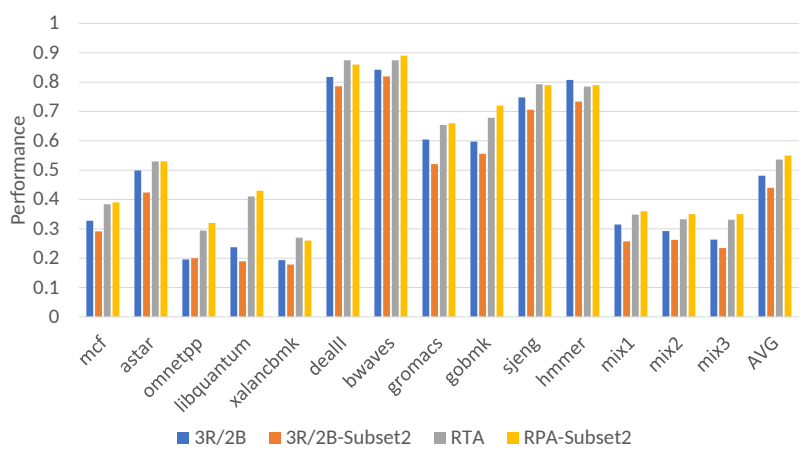

Figure 7: Performance comparison with and without rank subsetting for 3R/2B and for Ranked Alternation (RTA and RPA).

Figure 8 shows the performance of 4-way rank subsetting and 2-way rank subsetting with ranked alternation. With 4-way rank subsetting, the time between two accesses to the same rank is now 18 cycles and 55 for two accesses to the same bank. For 4-way rank subsetting, we use RTA with 18 cycle turns, and for 2-way rank subsetting, we use RPA with 10 cycle turns. The longer turn length with 4-way rank subsetting and the resulting longer data transfer time cause a degradation relative to the RTA without rank subsetting. Therefore, the higher parallelism provided by 2-way rank subsetting hits a sweet spot and even higher parallelism is detrimental.

\subsection{Overall Performance}

Finally, to set the performance of RTA in context, we show the primary design points in Figures 9 and 10. RTA improves upon $3 \mathrm{R} / 2 \mathrm{~B}$ by $11 \%$; adding rank subsetting takes the performance improvement up to $14 \%$. All of these techniques are hardware-based and require no additional support from the OS. These figures also show the performance benefit if there was additional OS support, as proposed by Shafiee et al. [12], to map each VM to its own banks (bank partitioning) or its own ranks (rank partitioning). We show 


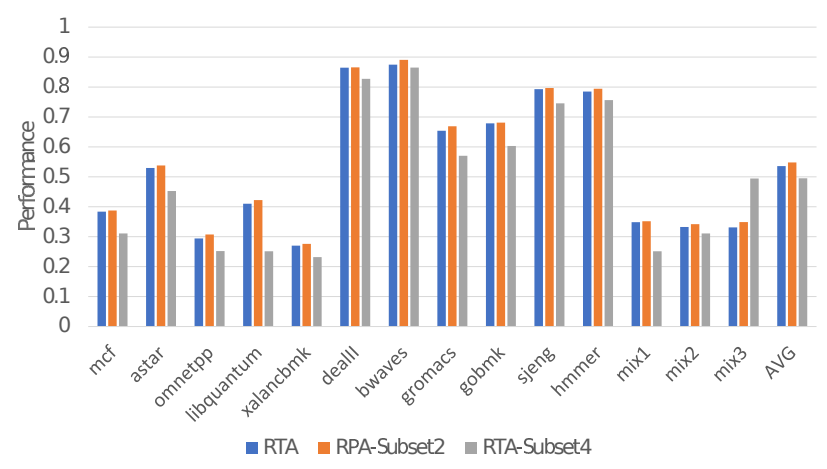

Figure 8: Performance comparison between 4-way and 2way rank subsetting with ranked alternation.

that our hardware-based techniques are now approaching the performance of a technique that relies on the OS for bank partitioning.

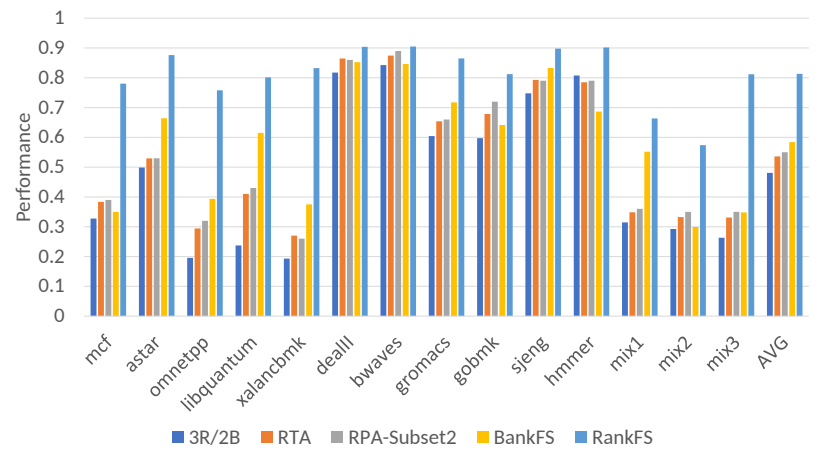

Figure 9: Overall performance of RTA, 3R/2B, RPA with 2way rank subsetting, Fixed Service controller with bank partitioning, and Fixed Service controller with rank partitioning.

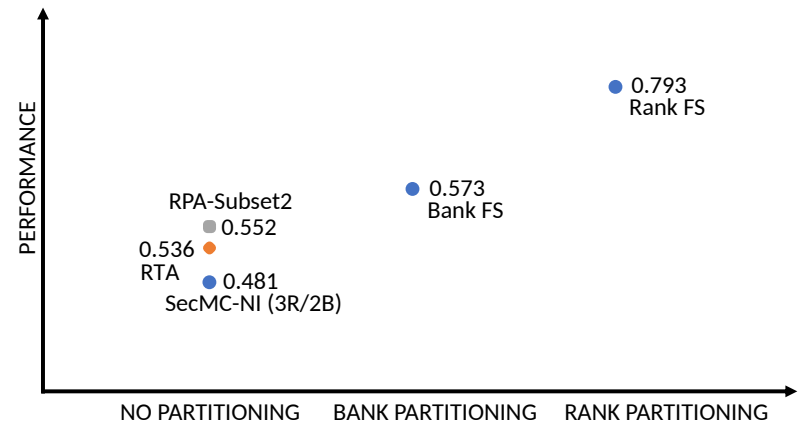

Figure 10: Overall performance of policies organized by OS support.

\section{CONCLUSIONS}

This paper introduces new scheduling strategies for the memory controller to improve performance while eliminating memory timing channels. The state-of-the-art scheduler 3R/2B uses relatively long turns that offer more issue slots per turn than can be exploited. Since most applications have low levels of MLP, we employ shorter turns and rely on scheduler constraints to prevent conflicts. The result is a scheduler, RTA, that improves performance by $11 \%$. This improvement grows to $14 \%$ when the memory system is augmented with rank subsets and an adapted scheduler, RPA. The new schedulers take a step forward in reducing the overheads introduced by leakage-free memory controllers without relying on support from the OS.

\section{ACKNOWLEDGMENT}

We thank the anonymous reviewers for many helpful suggestions. This work was supported in parts by NSF grants CNS-1302663, CNS-1423583, and CNS-1718834.

\section{REFERENCES}

[1] J. Ahn, N. Jouppi, and R. S. Schreiber. 2009. Future Scaling of Processor-Memory Interfaces. In Proceedings of SC.

[2] N. Chatterjee, R. Balasubramonian, M. Shevgoor, S. Pugsley, A. Udipi, A. Shafiee, K. Sudan, M. Awasthi, and Z. Chishti. 2012. USIMM: the Utah SImulated Memory Module. Technical Report. University of Utah. UUCS-12-002.

[3] Wind Company. 2007. Wind River Simics Full System Simulator. http://www.windriver.com/products/simics/

[4] D. Evtyushkin, R. Riley, N. Abu-Ghazaleh, and D. Ponomarev. 2018. BranchScope: A New Side-Channel Attack on Directional Branch Predictor. In Proceedings of ASPLOS.

[5] Casen Hunger, Mikhail Kazdagli, Ankit Rawat, Sriram Vishwanath, Alex Dimakis, and Mohit Tiwari. 2015. Understanding Contention-driven Covert Channels and Using Them for Defense. In Proceedings of HPCA.

[6] A. Jaleel. 2018 (retrieved). SPEC CPU2006 Memory Characterization. http://www.jaleels.org/ajaleel/workload/.

[7] JEDEC. 2012. JESD79-4: FEDEC Standard DDR4 SDRAM.

[8] P. Kocher, D. Genkin, D. Gruss, W. Haas, M. Hamburg, M. Lipp, S. Mangard, T. Prescher, M. Schwarz, and Y. Yarom. 2018. Spectre Attacks: Exploiting Speculative Execution. https://spectreattack.com/spectre.pdf.

[9] KT Nguyen. 2016. Introduction to Cache Allocation Technology in the Intel Xeon Processor E5 v4 Family. https://software.intel.com/en-us/articles/introduction-to-cache-allocation-technology.

[10] Thomas Ristenpart, Eran Tromer, Hovav Shacham, and Stefan Savage. 2009. Hey, You, Get Off of My Cloud: Exploring Information Leakage in Third-party Compute Clouds. In Proceedings of the 16th ACM conference on Computer and Communications Security. 199-212.

[11] D. Sanchez. 2018 (retrieved). ZSim Validation Data. http://zsim.csail.mit.edu/validation/time_series/.

[12] A. Shafiee, A. Gundu, M. Shevgoor, R. Balasubramonian, and M. Tiwari. 2015. Avoiding Information Leakage in the Memory Controller with Fixed Service Policies. In Proceedings of MICRO.

[13] J. Stuecheli. 2013. POWER8 Processor.

[14] Yao Wang, Andrew Ferraiuolo, and G. Edward Suh. 2014. Timing Channel Protection for a Shared Memory Controller. In Proceedings of HPCA.

[15] Y. Wang, B. Wu, and G.E. Suh. 2017. Secure Dynamic Memory Scheduling Against Timing Channel Attacks. In Proceedings of HPCA.

[16] Zhenghong Wang and Ruby B Lee. 2007. New Cache Designs for Thwarting Software Cache-based Side Channel Attacks. In Proceedings of ISCA.

[17] Zhenghong Wang and Ruby B Lee. 2008. A Novel Cache Architecture with Enhanced Performance and Security. In Proceedings of MICRO. 83-93.

[18] Z. Zhang, Z. Zhu, and X. Zhand. 2000. A Permutation-Based Page Interleaving Scheme to Reduce Row-Buffer Conflicts and Exploit Data Locality. In Proceedings of MICRO.

[19] H. Zheng, J. Lin, Z. Zhang, E. Gorbatov, H. David, and Z. Zhu. 2008. Mini-Rank: Adaptive DRAM Architecture For Improving Memory Power Efficiency. In Proceedings of MICRO. 